\title{
Conference Calendar
}

Audiology r Neuro Otology

12.11.-

15.11.1997

Berlin

Germany

1.12.-5.12.1997 San Diego California

USA

6.12.1997 Haifa

Israel

1.2.-6.2.1998 Courchevel

France

11.3.-12.3.1998 Oldenburg

Germany

19.3.-22.3.1998 Bad Kissingen

Germany

5.4.-9.4.1998 Nottingham

UK

2.4.-5.4.1998 Los Angeles California

USA

2nd International Laser Course on Otolaryngology, Head \& Neck Surgery

Fall Meeting of the Acoustical Society of America

24th International Congress of the Neurootological and Equilibriometric Society

GEM098

2nd European Otological and Neurootological Winter

Symposium

1. Jahrestagung der Deutschen Gesellschaft fiir Audiologie

XXVth International Congress of the Neurootological and Equilibriometric Society

18th European Instructional Course on Tinnitus and Its Management

10th Annual AAA Conference 'Catch a Rising Star'

Information: Frau Amdt, Sekretariat,

HNO Klinik, Universitätsklinikum

Benjamin Franklin, FU-Berlin,

Hindenburgdamm 30,

D-12200 Berlin (Germany)

Tel. (+49-30) 84452440 ,

Fax (+49-30) 84454460

Information: Acoustical Society of America, 500 Sunnyside Blvd., Woodbury, NY 11797

(USA), Tel. (+1-516)576-2357, Fax (+1-516) 576-2377 
Information: Prof. L. Podoshin, Department of Otolaryngology, Head \& Neck Surgery, Bnaí Zion Medical Center, Faculty of Medicine-Technion, P.O. Box 4940, Haifa 31048 (Israel) Information: Ms Sofie Mydoodt, IFOS/MISA/NKO, Oosterveldlaan 24, B-2610Wilrijk (Belgium), Tel. (+32-3) 44335 78, Fax (+32-3) 4433611

Information: Frau Karin Bramstedt,

AG Medizinische Physik,

Universität Oldenburg,

D-26111 Oldenburg (Germany),

Tel. (+49-441) 9706-470,

Fax (+49-441)798-3698,

E-mail: bramstedt@gst.uni-oldenburg.de

Information: C.F. Claussen, Neurootological Reseach Institute, Kurhausstrasse 12, D-97688 Bad Kissingen (Germany), Fax (+49-971) 68637

Information: Mrs E. Moxon, Ctr. for Cont. Prof. Development, University Park, Nottingham

NG7 2RD (UK), Fax (+44-115) 9513722

Information: Dennis Van Vliet, Chair of Meeting, University Park, Nottingham NG7 2RD (UK), Tel. (+1-714)579-0717, Fax (+1-714) 579-7827, E-mail: mbfn77a@prodigy.com

KAIIGEH

E-Mail karger@karger.ch Fax+4161306 1234

$\mathrm{V}, \mathrm{tti} \Lambda \cdot / /, \mathrm{m}^{\mathrm{TM}}, \mathrm{V}, \mathrm{roP} \Gamma \mathrm{rh}$

C1997S. KargerAG, Basel

15.B.-16.5.1998 Milan

Italy

5.6.-7.6.1998 Iowa City

USA

7.6.-10.6.1998 Helsinki

Finland

8.6.-11.6.1998 Antalya

Turkey

14.6.-16.6.1998 'S Hertogenbosch

The Netherlands

2.7-4.7.1998 Toulouse

France

28.8.-2.9.1998 Sendai

Japan

30.8.-3.9.1998 Buenos Aires

Argentina

20.9.-23.9.1998 Würzburg

Germany

European Consensus Development Conference (ECDC) on Neonatal Hearing Screening

Vllth Symposium on Cochlear Implants in Children

7th International Congress of Pediatric ORL

International Conference on Otology and Otoneurology Towards 2000's: Horizons of

Investigation and Treatment

4th European Symposium on Paediatric Cochlear Implantation 
2nd International Symposium on Minimally Invasive Technique and Image Guided Surgery in ENT and Skull Base Surgery

International Conference on Biomagnetism (BIOMAG98)

24th International Congress of Audiology

XXth Ordinary Meeting of the Barany Society

Information: F. Grandori, Centre of Biomedical Engineering, Polytechnic of Milan, Piazza

Leonardo da Vinci 32, 1-20133 Milan (Italy), Fax (+39-2)23 9933 60, E-mail:

ecdc@elet.polimi.it

Information: B. Gantz,

University of Iowa Hospitals and Clinic,

Department of ORL/HNS,

200 Hawkins Drive, E230 GH,

Iowa City, IA 52242-1078 (USA),

$\operatorname{Fax}(+1-319) 3563967$

Information: Prof. Pekka Karma, Department of ORL. Helsinki University Central Hosp., FIN00290 Helsinki (Finland), Fax (+35-8) 4712014

Information: O. Nuri Özgirgin, MD,

Baymdir Medical Center ENT Department.

TR-06520 Sögütözü Ankara (Turkey),

Tel. $(+90-312) 2879000$,

Fax (+90-312)285 07 33,

E-mail: Ozgirgin@neuron.ato.org.tr

Information: Prof. P. van den Broek, University Hospital Nijmegen, Department of ORL, P.O.

Box 9101 , NL-6500 HB Nijmegen (The Netherlands), Fax (+31-80) 540251

Information: Pr. Bernard Fraysse,

ENT Department,

Purpan University Hospital,

F-31059 Toulouse Cedex (France).

$\operatorname{Fax}(+33-61) 493644$

E-mail: fraysse@mail.cict.fr

Information: Secretariat Biomag98; Nobukazu Nakasato, Department of Neurosurgery, Tohoku University School of Medicine, 1-1 Seiryo-machi, Aboka-ku, Sendai 980-77 (Japan), Tel. (+8122) 717-7230, Fax (+81-22) 717-7233, E-mail: nak@gonryo.med.tohoku.ac.ip

Information: XXIV International Congress

of Audiology, c/o Congresos

Intemacionales S.A., Moreno 584, Piso 9

RA-1091 Buenos Aires (Argentina),

Tel. (+54-1) 342-3216/342-3283/342/3408,

Fax (+54-1) 331-0223/334 3811,

E-mail: conginte@mbox.servicenet.com.ar

Information: C.F. Claussen, Neurootological Research Institute, Kurhausstrasse 12, D-97688

Bad Kissingen (Germany). Fax (+49-971)68637

Conference Calendar

419

10.11.- 
14.11 .1998

Panama

Panama

16.4.-18.4.1999 Seoul

Korea

Spring 1999 Roma

Italy

June 2000 Marseille

France

2000 Berlin

Germany

26th Biennial Congress of Oto-Rhino-Laryngology, Head and Neck Surgery

2nd Congress of the Asia Pacific Symposium on Cochlear Implant and Related Sciences

3rd International Conference on Acoustic Neuroma Surgery

6th International Conference on Cholesteatoma and Mastoid Surgery

4th Quadrennial Congress European Federation for ORL, Head and Neck Surgery

Information: R. Crespo B,

Segundo Piso No. 227. Apartado 55-1376. Paitilla (Republic of Panama). Fax (+507-269) 1295

Information: C.-S. Kim, Department of ORL, Seoul National University College of Medicine, 28

Yongon-Dong, Chongno-Gu, Seoul 110-744 (Korea), Fax (+82-2) 7648700

Information: M. Sanna. Gruppo Otologico (Italy), Fax (+39-523) 712396

Information: A. Chays,

ORL Hôpital Nord, F-13915 Marseille,

Cedex 20 (France).

Fax (+33-4-91)96 2142

Information: ENT Department. Gentofte Hospital, University of Copenhagen, DK-2900 Hellerup (Denmark),

$\operatorname{Fax}(+45-3977) 7634$

420

Conference Calendar 